\title{
Upregulated IncRNA HCGI8 in Patients with Non-Alcoholic Fatty Liver Disease and Its Regulatory Effect on Insulin Resistance
}

\author{
Yu Xia \\ Yanxia Zhang \\ Huiyun Wang \\ Department of Health Comprehensive \\ Geriatrics, Yidu Central Hospital of \\ Weifang, Weifang, Shandong, People's \\ Republic of China
}

Purpose: Non-alcoholic fatty liver disease (NAFLD) is a disease associated with geneticenvironmental-metabolic stress, which severely damages the liver function of patients. This study aimed to explore the significance and probable functions of HCG18 in NAFLD.

Patients and Methods: The expression of HCG18 and miR-197-3p was tested by qRTPCR. The clinical signification of HCG18 was provided by the ROC curve and Pearson correlation. The corresponding mechanism was punctuated by the luciferase reporter assay and HFD-managed mice.

Results: HCG18 expression was higher in the patients with NAFLD than in controls and in individuals with HOMA-IR score $\geq 2.5$ than those with HOMA-IR score $<2.5$. HCG18 expression in NAFLD patients was related to BMI, HOMA-IR, ALT, FBG, TC, and TG. HCG18 showed satisfactory predictive accuracy in differentiating NAFLD patients and patients with HOMA-IR $\geq 2.5$. Besides, HCG18 had protective impacts on blood glucose and fat deposition but not on body weight. MiR-197-3p is a direct gene of HCG18, and a reverse correlation was found between miR-197-3p and HCG18. Furthermore, miR-197-3p regulated the influence of HCG18 on insulin resistance and lipid accumulation.

Conclusion: Increased levels of HCG18 might be an alternate indicator for NAFLD patients. The HCG18-miR-197-3p axis exerted effects on the progression of fat sedimentation and glucose disorder in NAFLD.

Keywords: HCG18 miR-197-3p, diagnosis, insulin resistance, lipid accumulation

\section{Introduction}

Non-alcoholic fatty liver disease (NAFLD) is the most common clinical liver disease, and its pathological changes have a broad coverage. ${ }^{1,2}$ Metabolic syndromes such as obesity, type 2 diabetes, hyperinsulinemia, and dyslipidemia are considered to be the main factors for the occurrence of NAFLD. ${ }^{3,4}$ Studies have shown that more than $90 \%$ of obese individuals have NAFLD and more than $70 \%$ have NAFLD. ${ }^{5}$ With the increase in the number of obese people, the prevalence of NAFLD has risen sharply, influencing about $25 \%$ of the total population worldwide. ${ }^{5}$ A recent meta-analysis revealed the prevalence of NAFLD in China was about $32.9 \%$ in $2018 .{ }^{6}$ NAFLD is characterized by marked inflammation and liver function impairment caused by intrahepatic fat accumulation, which can progress to hepatic fibrosis and cirrhosis. ${ }^{7}$ As a wide spectrum of clinical parameters, NAFLD may trigger fibrosis, cirrhosis, and advanced hepatocellular carcinoma. ${ }^{8}$ NAFLD has an important relationship with liver cancer
Correspondence: Yu Xia

Department of Health Comprehensive Geriatrics, Yidu Central Hospital of

Weifang, No. 4138, Linglongshan Road,

Weifang, Shandong, 262500, People's

Republic of China

Tel +86-536-3279993

Email xiayuvsrain@163.com 
and will become the main cause of liver cancer. Therefore, NAFLD has become a public health problem that cannot be ignored. ${ }^{9}$ Liver biopsy is the gold standard for the diagnosis of NAFLD, but its procedure is traumatic and cannot be widely carried out clinically. ${ }^{10}$ Therefore, screening specific indicators that are related to the diagnosis of NAFLD has become the focus of attention.

NAFLD is a common liver disease with high heritability and several molecular pathways lead to the pathogenesis of NAFLD. ${ }^{11,12}$ Owing to intensive research in recent years, it has been found that IncRNA plays an important regulatory role in much pathological development and physiological processes, and its expression in the liver and its relationship with liver diseases have increasingly become the focus of research. ${ }^{13,14}$ Besides, IncRNAs are quite stable in human serum and specific lncRNAs may serve as non-invasive predictors. ${ }^{15}$ The levels of lncRNA ARSR both in the serum are significantly elevated in NAFLD patients compared with the healthy controls. ${ }^{16}$ In a microarray of lncRNAs, 1735 lncRNAs are found aberrantly expressed in the NAFLD patients relative to that in healthy liver specimens. ${ }^{17}$ NAFLD in rodents is usually caused by a high-fat diet (HFD), which stimulates the excessive accumulation of fat in the liver and is commonly used to explore the mechanism underlying NAFLD. ${ }^{18}$ The levels of lncRNA NEAT1 are raised in the NAFLD mice models and it could facilitate lipid accumulation via miR-146a-5p. ${ }^{19}$ Several researchers have examined the importance of lncRNA HCG18 in liver diseases. For instance, in a previous subject of hepatocellular carcinoma, the expression of HCG18 is raised in the tumor tissues and accelerates tumor progression by targeting miR-214-3p. ${ }^{20}$ In another analysis of hepatocellular carcinoma, the expression of HCG18 is differently expressed. ${ }^{21}$ It seems that HCG18 may participate in the progression of liver dysfunction. Although some relationship between HCG18 and hepatocellular carcinoma has been unveiled, the mechanism of HCG18 involved in the development of NAFLD is still unsolved.

The purpose of this study was to investigate the expression level of serum HCG18 in patients with NAFLD and the diagnostic role of HCG18. The correlation with liver lipid metabolism indicators was analyzed to assess the significance of serum HCG18 expression in screening for liver metabolic abnormalities. The role and potential regulatory mechanism of HCG18 were explored in NAFLD mice by further studying its action as a miRNA sponge.

\section{Materials and Methods}

\section{Patient Collection}

We collected 101 healthy individuals and 116 NAFLD patients from Yidu Central Hospital of Weifang. According to a previous diagnosis of NAFLD, all NAFLD individuals were identified. ${ }^{22}$ The exclusion criteria were as follows: (1) patients with autoimmune hepatitis, viral hepatitis, or drug-induced liver disease, (2) individuals taking drugs that protect the liver, reduce fat or cause liver fat deposition, (3) people with secondary liver function damage, and (4) people with a history of alcoholism. This study was performed in accordance with the Declaration of Helsinki and the protocols of our design were approved by the Ethics Committee of Yidu Central Hospital of Weifang. Written informed consents were collected from all participants. The homeostasis model of assessment for IR index (HOMA-IR) was analyzed based on the clinical information collected from each individual. The blood samples were collected from all participants and the serum specimens were isolated for the following experiments.

\section{NAFLD Animal Model Construction}

Male C57Bl/6 mice (5 weeks old, weighing 17-19 g) were provided by Junke Bioengineering company (Nanjing, China) and were adopted in an atmosphere with humidity of $30-70 \%$ at $21 \pm 2^{\circ} \mathrm{C}$. To detect the function of HCG18, adaptive feeding mice were randomly divided into four groups, including the normal feed (ND) group, HFD group, small interfering negative control (si-NC) group, siHCG18 group. A further mechanism of HCG18 was revealed by the following three groups: si-HCG18, siHCG18 + antagomir NC, and si-HCG18 + miR-197-3p antagomir. A total of five mice were included in each group. The mice except those in the ND group were fed with high-fat food, containing $20 \%$ protein, $40 \%$ fat (soybean oil and lard), and $40 \%$ carbohydrate for 8 weeks. The mice in the ND group were fed with normal fat content (12\%) food for constantly 8 weeks. The protocols of diets for all mice were based on a previous study. ${ }^{23}$

The sequences of antagomir NC and miR-197-3p antagomir and lentiviruses expressing si-NC and siHCG18 were purchased from GenePharma (Shanghai, China). All mice in each group were injected with the corresponding reagent weekly for 8 weeks after HFD treatment. The weight of each mouse was recorded every 
7 days. The blood sample was injected and some characters were also detected after 8 weeks.

All procedures involving experimental animals were in line with the Guide for the Care and Use of Laboratory Animals of the Institute for Laboratory Animal Research. In addition, this study also was approved by the Ethics Committee of Yidu Central Hospital of Weifang.

\section{Quantitative PCR}

According to the manufacturer's instructions, a standard method was conducted to extract total RNA from serum via an extreme speed RNA extraction kit (HaiGene, Harbin, China). The concentration and purity of RNA samples were clarified by NanoDrop. The mixture of Probe One-Step RT-qPCR Kit (TAKARA, Tokyo, Japan) and total RNA was used to detect the levels of HCG18 on Roche LightCycler 480 (Roche, Basel, Switzerland). For miR-197-3p, the cDNA was amplified from total RNA using miRNA 1st strand cDNA synthesis kit (by stemloop) (Vazyme, Nanjing, China). A $2 \times$ miRNA qPCR master mix (Sangon Biotech, Shanghai, China) was applied in the quantitative expression of miR-197-3p on Roche LightCycler 480 (Roche, Basel, Switzerland). After amplification, a melting curve was generated to evaluate the efficiency of primer pairs at the end of each PCR cycle. And the amplification of only one product in qRT-PCR was confirmed by a melting curve analysis. The relative expression levels of HCG18 and miR-197-3p were determined by normalizing them to the internal genes $\beta$-actin and U6 respectively. ${ }^{24,25}$ The relative expression of HCG18 and miR-197-3p was calculated using the 2-DeltaDeltaCt method. As for mRNA expression of IL18, the detection kits and protocols were the same as the detection of HCG18 expression.

\section{Characteristics of C57BI/6 Mice}

For the intraperitoneal insulin tolerance test (IPITT), a total of $0.75 \mathrm{IU} / \mathrm{kg}$ insulin was injected intraperitoneally into the mice after 8-hours of fasting. The blood glucose concentration was detected 5 times every 30 minutes by a glucometer. And some other significant characters of mice were detected and recorded after all experiments.

\section{Luciferase Activity Report}

The transfected sequences, including miR-197-3p mimic, miR-197-3p inhibitor, and corresponding negative control (miR-NC) were purchased from GenePharma (Shanghai, China). The psiCHECK2 vectors carrying wide type (WT) and mutation (MUT) of HCG18 were from the Genecreate company (Wuhan, China). For detection of the relationship between IL18 and miR-197-3p, the carriers with WT-IL18 and MUT-IL18 were purchased from the same company. Different psiCHECK2 carriers were co-transfected with miR-197-3p mimic, miR-197-3p inhibitor, and miR-NC respectively. Then, the luciferase activity was verified using a dual-luciferase reporter assay system (Promega, Madison, WI, USA).

\section{Statistical Analysis}

The differences between two groups such as the HOMAIR $<2.5$ group and HOMA-IR $\geq 2.5$ group were calculated by the Student's $t$-test on SPSS software. Pearson correlation was conducted to investigate the relationship between HCG18 and parameters of NAFLD patients. The predictive value of HCG18 was unveiled by the area under the curve (AUC) of receiver operator characteristic (ROC). The comparison among four groups or six groups was analyzed by one-way ANOVA on the GraphPad software.

\section{Results}

\section{Baseline Features of Subjects}

We collected the clinical data of patients after confirming their inclusion in the study. The NAFLD group consisted of 60 females and 56 males with an average age of $49.80 \pm$ 9.74 years old. After analysis, the age and gender in the NAFLD group had no significant difference from those features in the healthy group (Table $1, P>0.05$ ). The levels of BMI, HOMA-IR, alanine transaminase (ALT), FBG, total cholesterol (TC), and triglycerides (TG) were elevated in the NAFLD group compared with healthy individuals (Table $1, P<0.01$ ).

\section{HCG 18 Levels in NAFLD Patients}

The concentration of HCG18 in serum samples from NAFLD patients was assessed to reveal the expression of HCG18 in NAFLD patients. Compared with the levels of HCG18 in the control group, HCG18 was highly expressed in the NAFLD group (Figure 1A, $P<0.001$ ). Furthermore, in the group of NAFLD, the patients were divided into two groups based on the values of HOMA-IR. There were 45 patients in the HOMA-IR $<2.5$ group and 71 patients in the HOMA-IR $\geq 2.5$ group. The expression of HCG18 was raised in the HOMA-IR $\geq 2.5$ patients relative to HOMAIR $<2.5$ patients (Figure 1B, $P<0.001$ ). 
Table I Clinical Features of Subjects

\begin{tabular}{|c|c|c|c|}
\hline Parameters & Healthy Individuals $(n=10 I)$ & NAFLD Group $(n=116)$ & $P$ value \\
\hline Gender (Female/ Male) & $52 / 49$ & $60 / 56$ & 0.972 \\
\hline Age (years) & $48.54 \pm 9.69$ & $49.80 \pm 9.74$ & 0.343 \\
\hline BMI $\left(\mathrm{kg} / \mathrm{m}^{2}\right)$ & $21.92 \pm 3.00$ & $27.84 \pm 2.98$ & $<0.001$ \\
\hline HOMA-IR & $1.22 \pm 0.09$ & $3.62 \pm 2.51$ & $<0.001$ \\
\hline ALT (IU/I) & $22.16 \pm 2.09$ & $39.58 \pm 6.19$ & $<0.001$ \\
\hline FBG $(\mathrm{mg} / \mathrm{dl})$ & $90.63 \pm 13.18$ & $108.70 \pm 35.40$ & $<0.001$ \\
\hline $\mathrm{TC}(\mathrm{mg} / \mathrm{dl})$ & $178.94 \pm 23.53$ & $196.11 \pm 46.84$ & 0.001 \\
\hline TG (mg/dl) & $106.62 \pm 15.33$ & $|30.6| \pm 38.00$ & $<0.001$ \\
\hline
\end{tabular}

Abbreviations: NAFLD, nonalcoholic fatty liver disease; BMI, body mass index; HOMA-IR, homeostasis model assessment insulin resistance; ALT, alanine transaminase; FBG, fasting blood glucose; TC, total cholesterol; TG, triglycerides.

\section{Correlation Between HCGI8 and NAFLD}

Through the Pearson correlation, the relationship between HCG18 expression and NAFLD was uncovered (Table 2). The result demonstrated that the HCG18 was pertinent to the BMI $(\mathrm{r}=0.604$, $P<0.001)$, HOMA-IR $(\mathrm{r}=0.717, P<0.001)$, ALT $(\mathrm{r}=0.631, P<0.001)$, FBG $(\mathrm{r}=0.726, P<0.001)$, TC $(\mathrm{r}=0.802, P<0.001)$, and TG $(\mathrm{r}=0.634, P<0.001)$. However, no relationships were found between HCG18 and gender $(\mathrm{r}=0.058, P=0.533)$ and age $(\mathrm{r}=0.008$, $P=0.929)$.

\section{Predictive Significance of HCGI 8 for NAFLD Patients}

The levels of HCG18 were identified as a promising prognostic indicator in differentiating NAFLD patients from the healthy group by the result of ROC which showed that the AUC was 0.934 (Figure 2A). More importantly, Figure 2B illustrated that the AUC of HCG18 was 0.881 which showed the levels of HCG18 could distinguish NAFLD patients with high insulin resistance from patients with NAFLD $<2.5$.

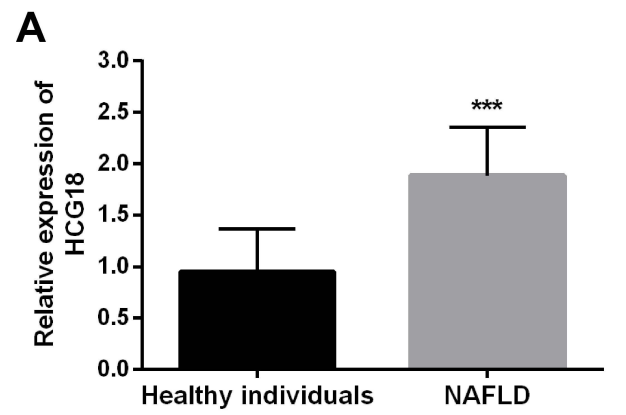

Table 2 Correlation Between IncRNA HCGI8 and Clinical Characteristics

\begin{tabular}{|l|c|c|}
\hline Characteristics & $\begin{array}{c}\text { Correlation with IncRNA } \\
\text { HCG I8 (r) }\end{array}$ & P-value \\
\hline Gender (Female/ & 0.058 & 0.533 \\
Male) & 0.008 & \\
Age (years) & 0.604 & 0.929 \\
BMI (kg/m²) & 0.717 & $<0.001$ \\
HOMA-IR & 0.631 & $<0.001$ \\
ALT (IU/l) & 0.726 & $<0.001$ \\
FBG (mg/dl) & 0.802 & $<0.001$ \\
TC (mg/dl) & 0.634 & $<0.001$ \\
TG (mg/dl) & & $<0.001$ \\
\hline
\end{tabular}

Abbreviations: NAFLD, nonalcoholic fatty liver disease; BMI, body mass index; HOMA-IR, homeostasis model assessment insulin resistance; ALT, alanine transaminase; FBG, fasting blood glucose; TC, total cholesterol; TG, triglycerides.

\section{Influence of HCGI8 on HFD Mice}

In the serum specimens of HFD mice, the expression of HCG18 was increased while si-HCG18 could successfully reduce the levels of HCG18 (Figure 3A, $P<0.001$ ). In addition, some characteristics were detected to provide the function of HCG18 on NAFLD, including body weight, insulin resistance level, and lipid metabolism. The analysis of body weight propounded that HFD management could elevate body weight

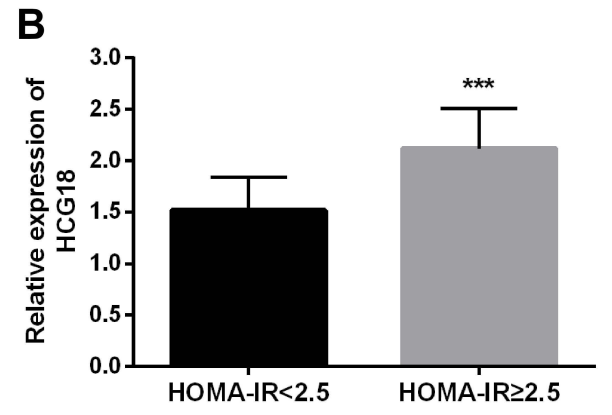

Figure I The expression of HCGI8 of $(\mathbf{A})$ all clinical patients and $(\mathbf{B})$ patients with HOMA-IR $\geq 2.5$. $* * * P<0.00$ I. 
A

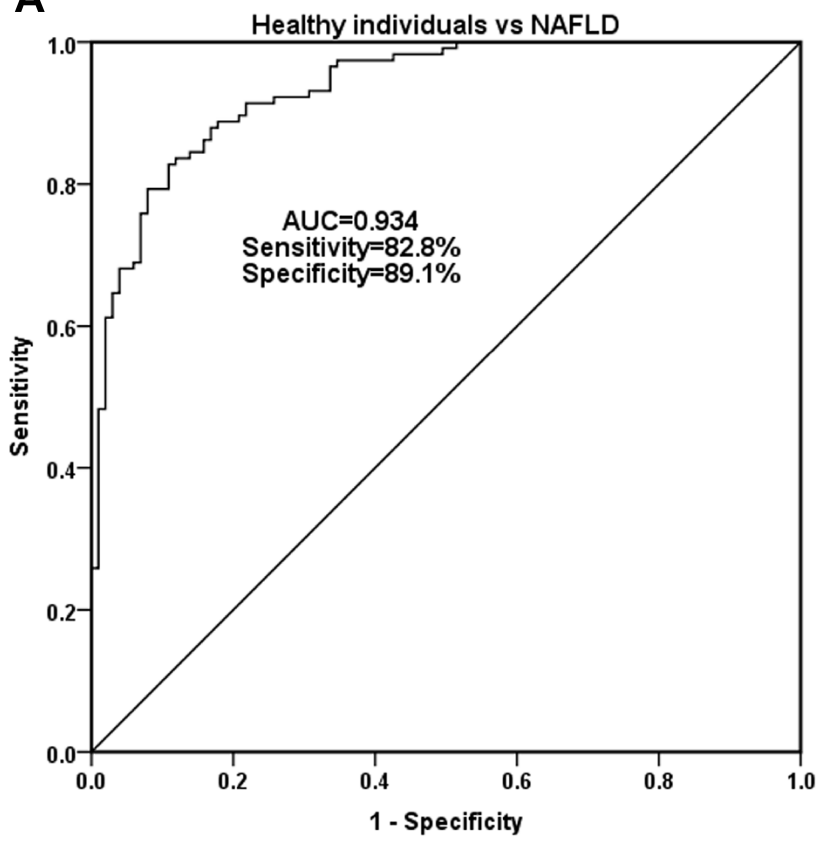

B

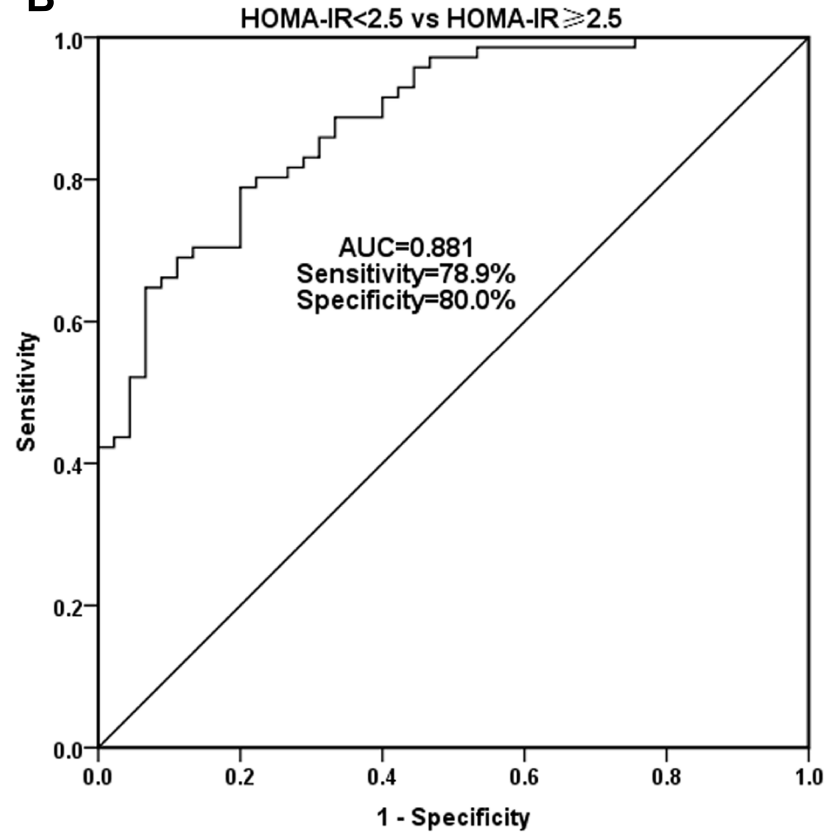

Figure 2 Diagnostic values of HCGI8 on the (A) NAFLD patients and (B) patients with HOMA-IR $\geq 2.5$.

A

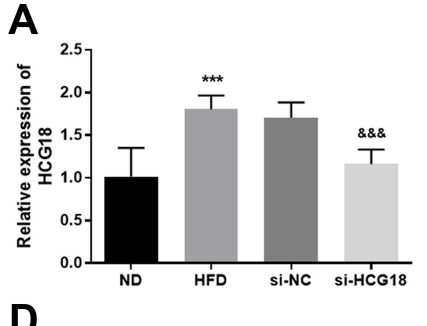

D

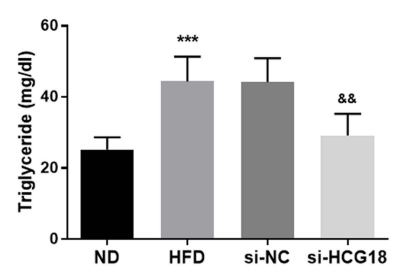

B

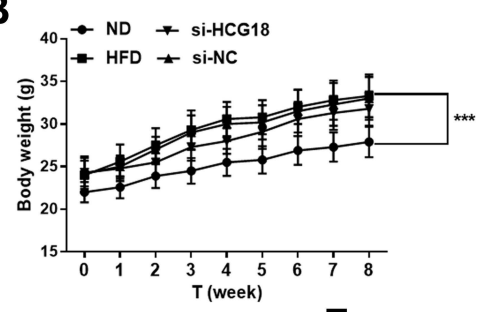

E

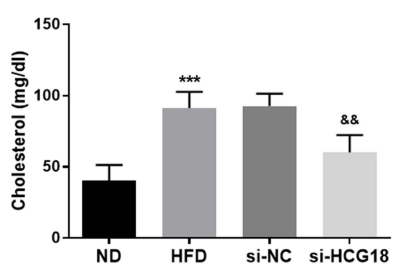

$\mathbf{F}$

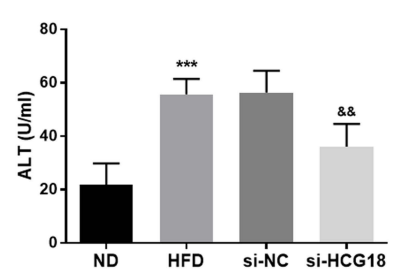

C

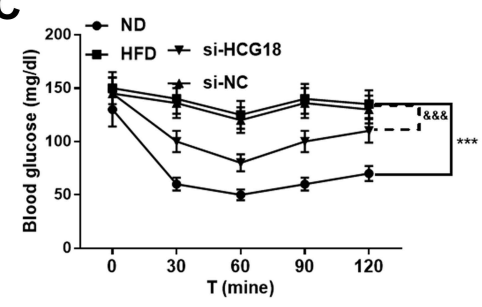

G

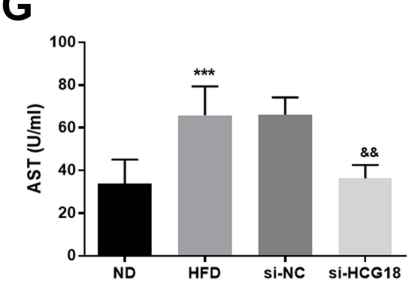

Figure 3 The roles of HCGI8 on the NAFLD animal models. (A) The reduced expression of HCG 18 in HFD mice was reversed by the si-HCG 18 . The impacts of HCG I8 on (B) body weight, (C) blood glucose, (D) triglyceride, (E) cholesterol, (F) ALT, and (G) AST. ${ }^{* * * P}<0.00$ I, relative to ND group; ${ }^{\& \&} P<0.0 \mathrm{I},{ }^{\& \& \&} P<0.00 \mathrm{I}$, relative to HFD group.

(Figure 3B, $P<0.001$ ), whereas, the silenced HCG18 had no significant impacts on the increased body weight (Figure 3B, $P>0.05)$. Regarding insulin resistance, HFD might contribute to the enhancement of blood glucose in mice, however, interference with HCG18 ameliorated the levels of blood glucose (Figure 3C, $P<0.001$ ). Besides, the concentration of TG, cholesterol, ALT, and AST was elevated in the HFD group while the suppression of HCG18 mitigated the increased tendencies of these indicators (Figure 3D-G, $P<0.01$ ).

\section{miR-197-3p Serves as a ceRNA of HCG 18}

Further study about the ceRNA of HCG18 was conducted to clarify the underlying mechanism. The finding of the predictive target was exhibited in Figure 4A, which proposed that the binding sites between HCG18 and miR-1973p. The miR-197-3p inhibitors reduced the luciferase activity, while the overexpression of miR-197-3p raised the activity, which validated the predictive results 
A

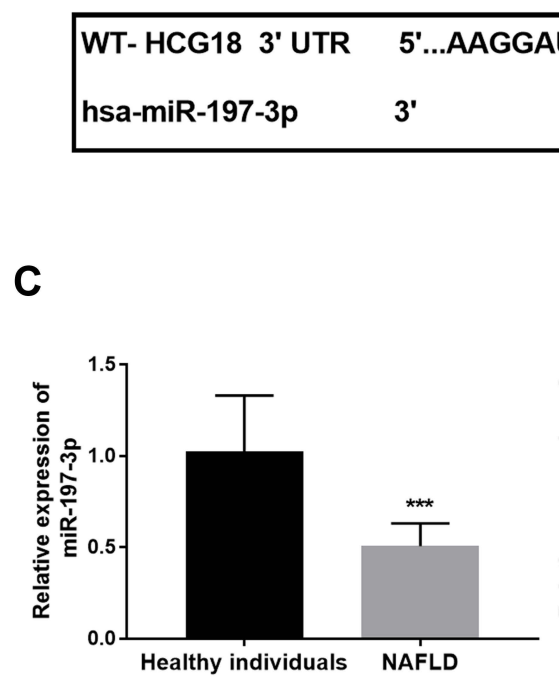

D

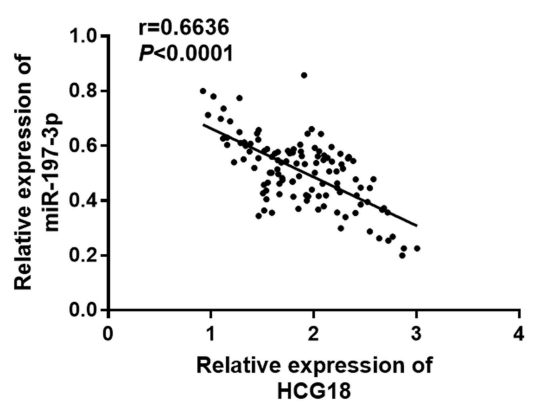

B

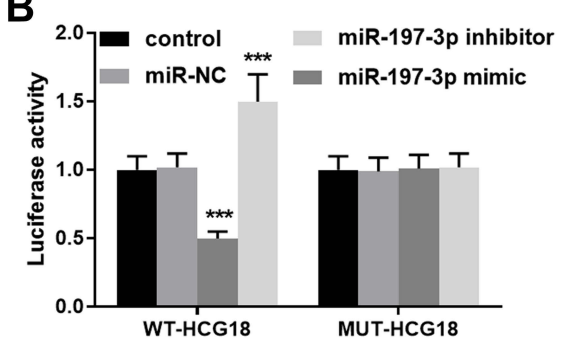

E

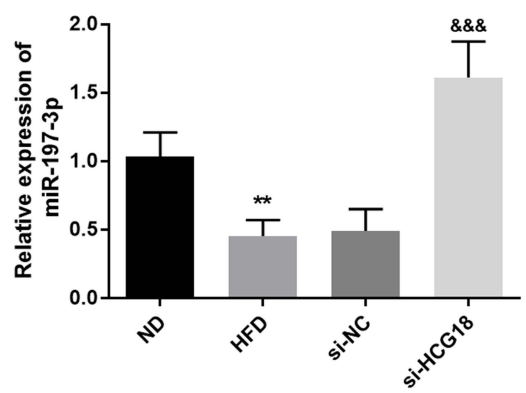

Figure 4 (A) The binding sites between HCGI8 and miR-197-3p. (B) The exhibition of luciferase report. (C) The reduced expression of miR-197-3p in the serum samples of NAFLD patients. (D) The levels of HCGI8 were inverse proportion to miR-197-3p. (E) The si-HCGI8 reversed the expression of miR-197-3p in the HFD mice. ${ }^{* * P}<0.0 \mathrm{I}$, $* * * P<0.00 \mathrm{I}$, relative to control group, healthy individuals, or ND group; ${ }^{\& \& \&} p<0.00 \mathrm{I}$, relative to HFD group.

(Figure 4B, $P<0.001$ ). Moreover, the expression of miR$197-3 p$ in the serum of NAFLD patients was accessed by qRT-PCR, and its correlation with HCG18 was also analyzed. As shown in Figure 4C, the expression of miR-197$3 p$ was lessened in the serum from NAFLD patients when compared with the healthy group $(P<0.001)$. The Pearson result reflected the negative correlation between miR-197$3 p$ expression and HCG18 levels in NAFLD patients (Figure 4D, $\mathrm{r}=0.6636, P<0.0001$ ). More importantly, in the HFD-managed mice, the Si-HCG18 inhibited the reduced miR-197-3p levels caused by HFD treatment (Figure 4E, $P<0.01$ ), further identifying the relationship between HCG18 and miR-197-3p.

\section{miR-197-3p Mediated the Impacts of HCGI 8 on HFD Mice}

The co-transfection experiments were conducted in the HFD mice and the finding of qRT-PCR indicated that the expression of miR-197-3p was suppressed by the co-transfection of si-HCG18 and miR-197-3p antagomir (Figure 5A, $P<0.001)$. The body weight in the si-HCG18 group was not influenced by the treatment of si-HCG18 and miR-1973 p (Figure 5B, $P>0.05$ ). The co-transfection of si-HCG18 and miR-197-3p antagomir reversed the levels of glucose caused by si-HCG18 in the HFD-triggered mice models (Figure 5C, $P<0.001$ ). Importantly, the miR-197-3p was proved to mediate the function of HCG18 on lipid metabolism by the findings the intervention of miR-197-3p reversed the ascended levels of TG, cholesterol, ALT, and AST (Figure 5D-G, $P<0.01$ ).

\section{ILI 8 Was a Target of miR-197-3p}

The putative sites between IL18 and miR-197-3p were shown in Figure 6A. Luciferase reporter assay elucidated that miR-197-3p mimic could suppress the luciferase activities and miR-197-3p inhibitor enhanced the luciferase activities in the WT-IL18 group (Figure 6B, $P<0.001)$. Besides, the mRNA expression of IL18 was elevated in the HFD-fed mice compared with the ND group (Figure 6C, $P<0.001$ ). Besides, si-HCG18 interference inhibited the expression of IL18, whereas, the cotransfection of si-HCG18 and miR-197-3p antagomir reversed the decreased mRNA expression of IL18 (Figure 6C, $P<0.001$ ).

\section{Discussion}

NAFLD is a chronic progressive liver disease, which belongs to simple hepatic steatosis and may develop into severe liver cirrhosis. ${ }^{26}$ Therefore, it is important to understand the pathogenesis of NAFLD and develop effective treatment methods. LncRNA plays a significant role in various physiological and pathological processes. ${ }^{27}$ At 

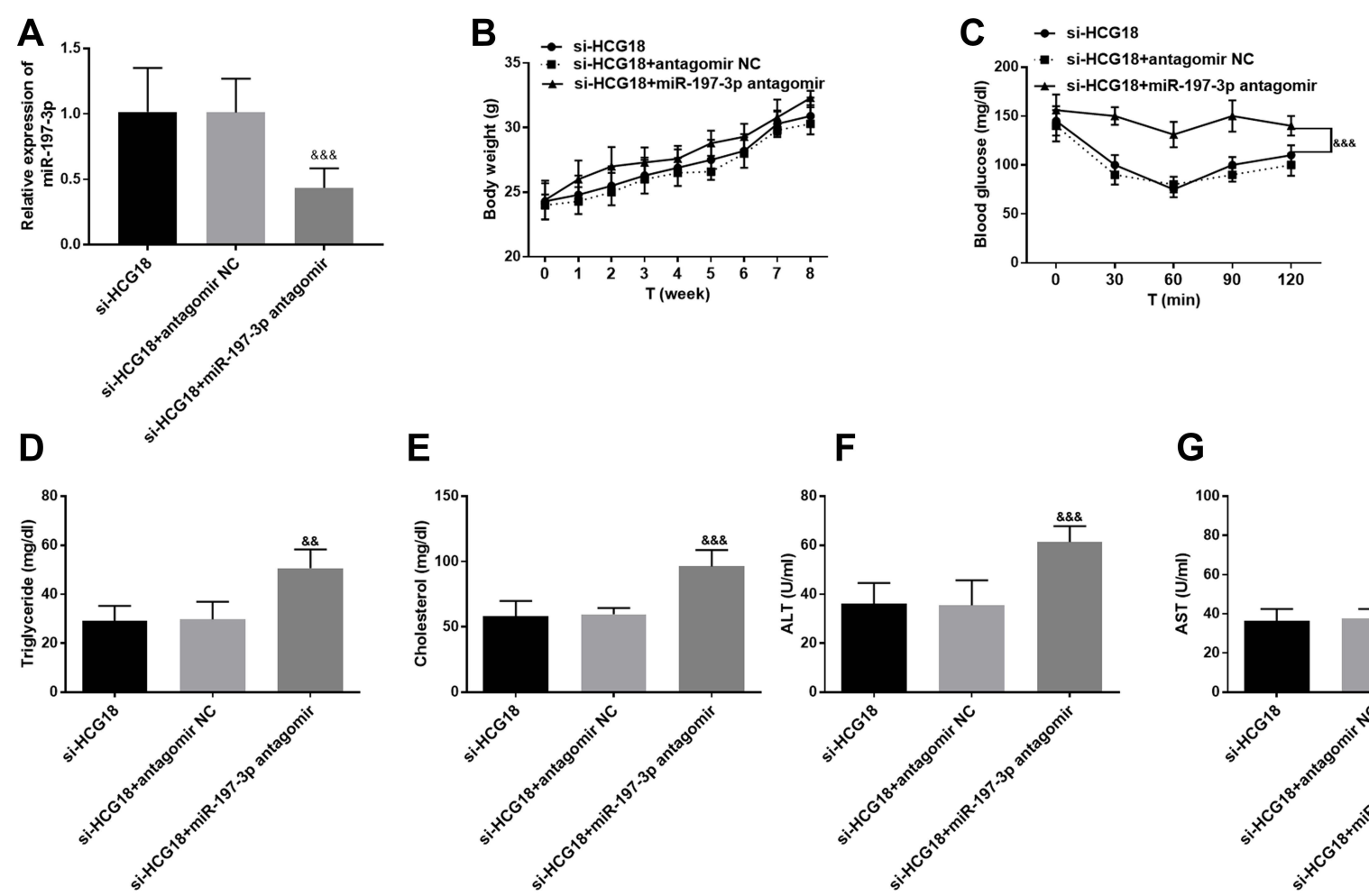

E

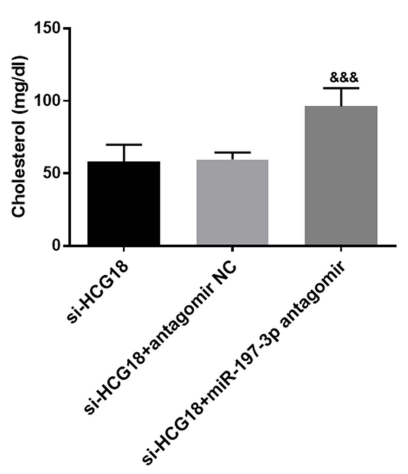

$\mathbf{F}$

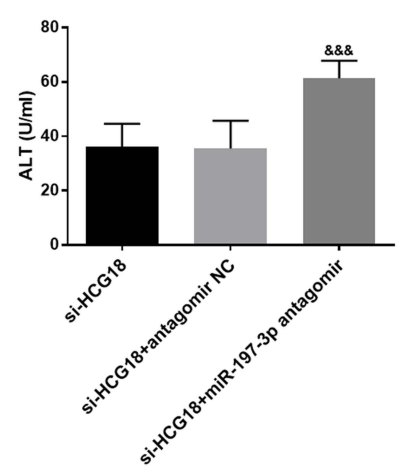

G

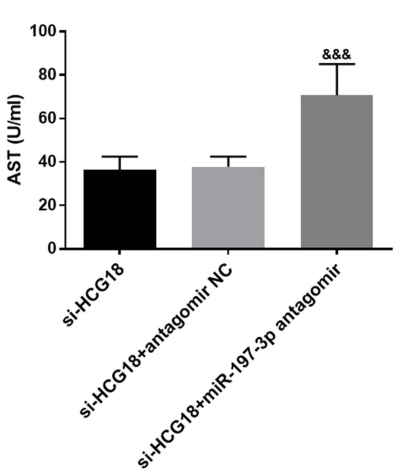

Figure 5 (A) MiR-197-3p antagomir inhibited the increased expression of miR-197-3p induced by si-HCGI8 in HFD mice. MiR-197-3p suppressed the impacts of HCG I8 on (B) body weight, (C) blood glucose, (D) triglyceride, (E) cholesterol, (F) ALT, and (G) AST. ${ }^{\& \&} P<0.01$, ${ }^{\& \& \&} P<0.00$ I, relative to HFD group.

A

WT-IL18 3' UTR 5'...CCAGCCUGACCAACAUGGUGAAA hsa-miR-197-3p 3' CGACCACCUCUUCCACCACUU

\section{B}

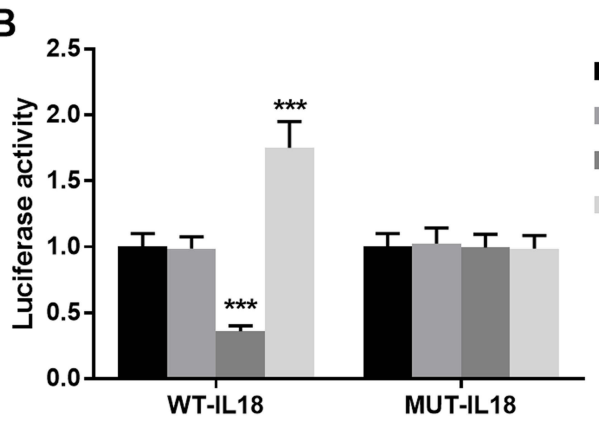

C

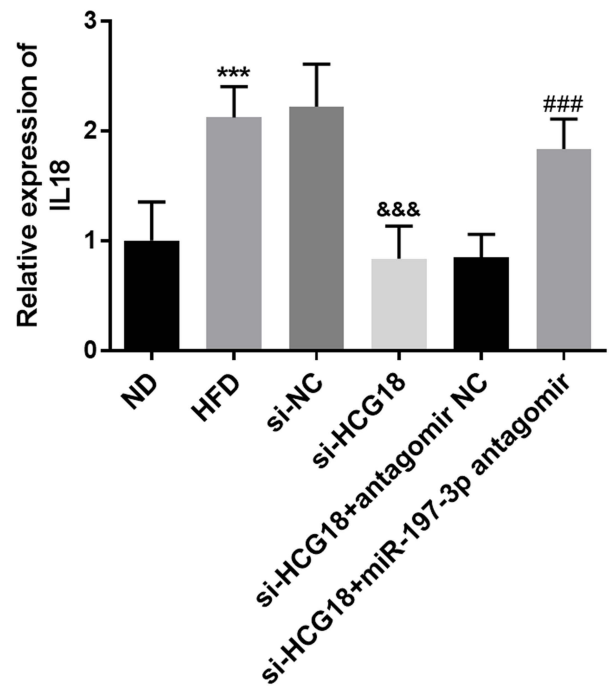

Figure 6 (A) The complementary sites between ILI8 and miR-197-3p. (B) The finding of luciferase reporter assay. (C) The expression of HCGI8 and miR-197-3p could

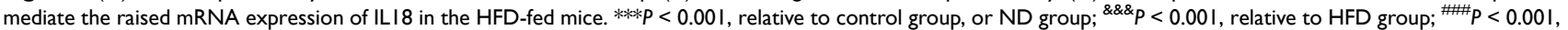
relative to si-HCGI8 group. 
present, IncRNA imbalance has been discovered in the liver of obese mice and severe NAFLD patients induced by HFD or genetic factors, which suggests the potential role of lncRNA in the development of liver dysfunction caused by diet regulation. ${ }^{28}$ Recent studies have shown that various lncRNAs are identified as important regulatory factors in lipid metabolism, fat factor expression, and fat formation. ${ }^{13,29}$ In addition, lncRNA is considered as a potential therapeutic target and biomarker for human liver diseases including NAFLD. ${ }^{30,31}$ Therefore, in this study, we focused on the abnormal expression of HCG18 and studied the regulation of HCG18 on lipid metabolism and insulin resistance of NAFLD by targeting miR-197-3p.

In our study, the expression of HCG18 was increased in the NAFLD patients, insisting that HCG18 might play a fundamental role in the NAFLD. Furthermore, compared to the HOMA-IR $<2.5$ group, the abundance of HCG18 expression was also found in the HOMA-IR $\geq 2.5$, indicating the levels of HCG18 might be associated with insulin resistance. This result was further clarified by demonstrating that the HCG18 was closely associated with the levels of BMI, HOMA-IR, ALT, FBG, TC, and TG. A microarray study about hepatocellular carcinoma patients finds that the expression of HCG18 is enriched in the tumor tissues compared with the adjacent healthy samples. ${ }^{21}$ LncRNA has become a candidate biological marker for a variety of diseases, especially as a noninvasive biomarker with special value in the prediction and diagnosis of liver diseases. ${ }^{15}$ The plasma lncRNA LeXis is identified as an alternative biomarker of NAFLD by the ROC curve, which indicates lncRNA has prognostic possibility. ${ }^{32}$ In hepatocellular carcinoma, LINC02518 may act as an indicator to ease the diagnosis of patients with hepatocellular carcinoma. ${ }^{33}$ In the present study, the diagnostic significance of HCG18 was also analyzed and the finding unveiled that HCG18 expression could discriminate NAFLD patients from healthy individuals and differentiate patients with HOMA-IR $\geq 2.5$ from HOMA-IR $<2.5$ group.

More and more studies have emphasized the role of lncRNA in gene regulation of liver function and diseases. Many liver pathologies related to NAFLD, including liver inflammation, structural damage, and lipid deposition, are regulated by one or more lncRNA. Blnc1 is found highly expressed in the NAFLD mice and participates in the regulation of insulin resistance in the progression of NAFLD. ${ }^{34}$ MEG3 is found to mediate lipid deposition of NAFLD by inhibiting the levels of miR-21. ${ }^{35}$ The function of HCG18 on mice models, such as bone loss and tumor xenografts has been examined by many investigators. ${ }^{36,37}$ Our data established NAFLD mice and found HFD was a trigger for the increased levels of HCG18, which further verified the clinical findings. Moreover, the absence of HCG18 could reverse the bad effects of HFD on weight, glucose, and lipid deposition, indicating disruption of HCG18 might have mitigated impacts on NAFLD.

The interaction between IncRNA and miRNA mediates several disorders, including NAFLD. A study written by Sun et al provides that NEAT1 has a progressive effect on the NAFLD by binding miR-140. ${ }^{38} \mathrm{H} 19$ might participate in the lipid metabolism of the NAFLD by regulating miR130a. Our result demonstrated that miR-197-3p might be a target of HCG18 in the mediation of NAFLD and the inverse association between them further underlined this finding. In gastric cancer, the relationship between HCG18 and miR-197-3p is identified and this pathway facilitates the development of gastric cancer. ${ }^{39}$ In a conclusion of miRNA array, miR-197-3p is lowly expressed in the NAFLD patients and related to the pericellular fibrosis in patients with steatohepatitis. ${ }^{40,41}$ In addition, miRNA might be a ceRNA of IncRNA, which plays an important role in the biological process and other vital activities. ${ }^{42}$ Our experiments confirmed that in the HFD-engendered mice, the knockdown of miR-197-3p reversed the amelioration of silenced HCG18 on blood glucose and fat accumulation, which also provided the underlying protective roles of miR-197-3p in NAFLD.

In the present study, IL18 was certified as a target gene of miR-197-3p. In hepatitis B virus infection, IL-18 is one of the possible target genes for miR-197-3p, which is consistent with our finding. ${ }^{43}$ In addition, we found the expression of IL18 was raised in the HFD-fed mice, which indicated that IL-18 might participate in the development of NAFLD. Besides, the alternation of HCG18 expression and miR-197-3p could change the mRNA expression of IL18. In a previous study, increased IL-18 expression has been observed in NAFLD, indicating Its role and importance as a biomarker of liver disease. ${ }^{44}$ Zhang et al report that the serum expression of IL18 in patients with liver cancer and fatty liver disease is significantly elevated, suggesting IL18 may act as an activator of liver diseases. ${ }^{45}$ Some limitations also exist in this study, for instance, the lack of liver tissue study and the NAFLD staging of experimental mice. 


\section{Conclusion}

Herein, the levels of HCG18 were at high levels and correlated with the expression of miR-197-3p. A close relationship between HCG18 and characteristics of NAFLD was identified and HCG was a diagnostic marker for NAFLD patients. MiR-197-3p was a crRNA of HCG18 and mediated the function of HCG18 on insulin resistance and fat accumulation.

\section{Disclosure}

The authors report no conflicts of interest in this work.

\section{References}

1. Lee J, Ha J, Jo K, et al. Male-specific association between subclinical hypothyroidism and the risk of non-alcoholic fatty liver disease estimated by hepatic steatosis index: Korea National Health and Nutrition Examination Survey 2013 to 2015. Sci Rep. 2018;8 (1):15145. doi:10.1038/s41598-018-32245-0

2. Cobbina E, Akhlaghi F. Non-alcoholic fatty liver disease (NAFLD) pathogenesis, classification, and effect on drug metabolizing enzymes and transporters. Drug Metab Rev. 2017;49(2):197-211. doi:10.1080/ 03602532.2017.1293683

3. Takai A, Kikuchi K, Ichimura M, et al. Fructo-oligosaccharides ameliorate steatohepatitis, visceral adiposity, and associated chronic inflammation via increased production of short-chain fatty acids in a mouse model of non-alcoholic steatohepatitis. BMC Gastroenterol. 2020;20(1):46. doi:10.1186/s12876-020-01194-2

4. Mundi MS, Velapati S, Patel J, Kellogg TA, Abu Dayyeh BK, Hurt RT. Evolution of NAFLD and its management. Nutr Clin Pract. 2020;35(1):72-84. doi:10.1002/ncp.10449

5. Qi Y, Wang W, Song Z, Aji G, Liu XT, Xia P. Role of sphingosine kinase in Type 2 diabetes mellitus. Front Endocrinol. 2020;11:627076. doi:10.3389/fendo.2020.627076

6. Zhou J, Zhou F, Wang W, et al. Epidemiological features of NAFLD from 1999 to 2018 in China. Hepatology. 2020;71(5):1851-1864. doi:10.1002/hep.31150

7. Schneider M, Benkert T, Solomon E, et al. Free-breathing fat and R (2) * quantification in the liver using a stack-of-stars multi-echo acquisition with respiratory-resolved model-based reconstruction. Magn Reson Med. 2020;84(5):2592-2605. doi:10.1002/mrm.28280

8. Chandrasekharan K, Alazawi W. Genetics of non-alcoholic fatty liver and cardiovascular disease: implications for therapy? Front Pharmacol. 2019;10:1413. doi:10.3389/fphar.2019.01413

9. Geh D, Manas DM, Reeves HL. Hepatocellular carcinoma in non-alcoholic fatty liver disease-A review of an emerging challenge facing clinicians. Hepatobiliary Surg Nutr. 2021;10(1):59-75. doi:10.21037/hbsn.2019.08.08

10. Kim TH, Jeong CW, Jun HY, et al. Accuracy of proton magnetic resonance for diagnosing non-alcoholic steatohepatitis: a meta-analysis. Sci Rep. 2019;9(1):15002. doi:10.1038/s41598-01951302-w

11. Sookoian S, Pirola CJ, Valenti L, Davidson NO. Genetic pathways in nonalcoholic fatty liver disease: insights from systems biology. Hepatology. 2020;72(1):330-346. doi:10.1002/hep.31229

12. Eslam M, George J. Genetic contributions to NAFLD: leveraging shared genetics to uncover systems biology. Nat Rev Gastroenterol Hepatol. 2020;17(1):40-52. doi:10.1038/s41575-019-0212-0

13. Sulaiman SA, Muhsin NIA, Jamal R. Regulatory Non-coding RNAs network in non-alcoholic fatty liver disease. Front Physiol. 2019;10:279. doi:10.3389/fphys.2019.00279
14. He Z, Yang D, Fan X, et al. The roles and mechanisms of lncRNAs in liver fibrosis. Int J Mol Sci. 2020;21(4):1482. doi:10.3390/ijms21041482

15. Di Mauro S, Scamporrino A, Petta S, et al. Serum coding and non-coding RNAs as biomarkers of NAFLD and fibrosis severity. Liver Int. 2019;39(9):1742-1754. doi:10.1111/liv.14167

16. Zhang M, Chi X, Qu N, Wang C. Long noncoding RNA lncARSR promotes hepatic lipogenesis via Akt/SREBP-1c pathway and contributes to the pathogenesis of nonalcoholic steatohepatitis. Biochem Biophys Res Commun. 2018;499(1):66-70. doi:10.1016/j. bbrc.2018.03.127

17. Sun C, Huang F, Yan W, Liu H, Wang C. [Expression profile of long non-coding RNA in non-alcoholic fatty liver disease]. Zhong Nan Da Xие Xие Bao Yi Xue Ban. 2017;42(7):741-748. Chinese. doi:10.11817/j.issn.1672-7347.2017.07.001

18. Zhou JP, Ren YD, Xu QY, et al. Obesity-induced upregulation of ZBTB7A promotes lipid accumulation through SREBP1. Biomed Res Int. 2020;2020:4087928. doi:10.1155/2020/4087928

19. Chen X, Tan XR, Li SJ, Zhang XX. LncRNA NEAT1 promotes hepatic lipid accumulation via regulating miR-146a-5p/ROCK1 in nonalcoholic fatty liver disease. Life Sci. 2019;235:116829. doi:10.1016/j.1fs.2019.116829

20. Zou Y, Sun Z, Sun S. LncRNA HCG18 contributes to the progression of hepatocellular carcinoma via miR-214-3p/CENPM axis. J Biochem. 2020;168(5):535-546. doi:10.1093/jb/mvaa073

21. Huang PS, Chang CC, Wang CS, Lin KH. Functional roles of non-coding RNAs regulated by thyroid hormones in liver cancer. Biomed J. 2021;44(3):272-284. doi:10.1016/j.bj.2020.08.009

22. Chalasani N, Younossi Z, Lavine JE, et al. The diagnosis and management of non-alcoholic fatty liver disease: practice guideline by the American Association for the Study of Liver Diseases, American College of Gastroenterology, and the American Gastroenterological Association. Hepatology. 2012;55(6):2005-2023. doi:10.1002/hep.25762

23. Sheng D, Zhao S, Gao L, et al. BabaoDan attenuates high-fat diet-induced non-alcoholic fatty liver disease via activation of AMPK signaling. Cell Biosci. 2019;9:77. doi:10.1186/s13578-0190339-2

24. Dongiovanni P, Meroni M, Baselli G, et al. PCSK7 gene variation bridges atherogenic dyslipidemia with hepatic inflammation in NAFLD patients. J Lipid Res. 2019;60(6):1144-1153. doi:10.1194/ jlr.P090449

25. Zhang $\mathrm{Z}$, Liu $\mathrm{X}, \mathrm{Xu} \mathrm{H}$, et al. Obesity-induced upregulation of miR-361-5p promotes hepatosteatosis through targeting Sirt1. Metabolism. 2018;88:31-39. doi:10.1016/j.metabol.2018.08.007

26. Ryu JS, Lee M, Mun SJ, et al. Targeting CYP4A attenuates hepatic steatosis in a novel multicellular organotypic liver model. J Biol Eng. 2019;13:69. doi:10.1186/s13036-019-0198-8

27. Wu Y, Zhou Y, Huan L, et al. LncRNA MIR22HG inhibits growth, migration and invasion through regulating the miR-10a-5p/NCOR2 axis in hepatocellular carcinoma cells. Cancer Sci. 2019;110 (3):973-984. doi:10.1111/cas.13950

28. Rohilla S, Awasthi A, Kaur S, Puria R. Evolutionary conservation of long non-coding RNAs in non-alcoholic fatty liver disease. Life Sci. 2021;264:118560. doi:10.1016/j.1fs.2020.118560

29. Ji E, Kim C, Kim W, Lee EK. Role of long non-coding RNAs in metabolic control. Biochim Biophys Acta Gene Regul Mech. 2020;1863(4):194348. doi:10.1016/j.bbagrm.2018.12.006

30. Bu FT, Wang A, Zhu Y, et al. LncRNA NEAT1: shedding light on mechanisms and opportunities in liver diseases. Liver Int. 2020;40 (11):2612-2626. doi:10.1111/liv.14629

31. Khalifa O, Errafii K, Al-Akl NS, Arredouani A. Noncoding RNAs in nonalcoholic fatty liver disease: potential diagnosis and prognosis biomarkers. Dis Markers. 2020;2020:8822859. doi:10.1155/2020/ 8822859

32. Park JG, Kim G, Jang SY, et al. Plasma long noncoding RNA LeXis is a potential diagnostic marker for non-alcoholic steatohepatitis. Life. 2020;10(10). doi:10.3390/life10100230 
33. Cui W, Huang J, Wang R, et al. Predictive value of a novel lncRNA LINC02518 in evaluating the prognosis of patients with hepatocellular carcinoma. Biomark Med. 2021;15(14):1277-1288. doi: 10.2217/bmm-2020-0795

34. Zhao XY, Xiong X, Liu T, et al. Long noncoding RNA licensing of obesity-linked hepatic lipogenesis and NAFLD pathogenesis. Nat Commun. 2018;9(1):2986. doi:10.1038/s41467-018-05383-2

35. Huang P, Huang FZ, Liu HZ, Zhang TY, Yang MS, Sun CZ. LncRNA MEG3 functions as a ceRNA in regulating hepatic lipogenesis by competitively binding to miR-21 with LRP6. Metabolism. 2019;94:1-8. doi:10.1016/j.metabol.2019.01.018

36. Che M, Gong W, Zhao Y, Liu M. Long noncoding RNA HCG18 inhibits the differentiation of human bone marrow-derived mesenchymal stem cells in osteoporosis by targeting miR-30a-5p/NOTCH1 axis. Mol Med. 2020;26(1):106. doi:10.1186/s10020-020-00219-6

37. Ma F, An K, Li Y. Silencing of long non-coding RNA-HCG18 inhibits the tumorigenesis of gastric cancer through blocking PI3K/ Akt pathway. Onco Targets Ther. 2020;13:2225-2234. doi:10.2147/ ott.s240965

38. Sun Y, Song Y, Liu C, Geng J. LncRNA NEAT1-MicroRNA-140 axis exacerbates nonalcoholic fatty liver through interrupting AMPK/ SREBP-1 signaling. Biochem Biophys Res Commun. 2019;516 (2):584-590. doi:10.1016/j.bbrc.2019.06.104

39. Niu W, Guo LY, Zhang JY, et al. E2F1-induced upregulation of IncRNA HCG18 stimulates proliferation and migration in gastric cancer by binding to miR-197-3p. Eur Rev Med Pharmacol Sci. 2020;24(19):9949-9956. doi:10.26355/eurrev_202010_23207
40. Estep M, Armistead D, Hossain N, et al. Differential expression of miRNAs in the visceral adipose tissue of patients with non-alcoholic fatty liver disease. Aliment Pharmacol Ther. 2010;32(3):487-497. doi:10.1111/j.1365-2036.2010.04366.x

41. Lan $\mathrm{X}, \mathrm{Wu} \mathrm{L}, \mathrm{Wu} \mathrm{N}$, et al. Long noncoding RNA lnc-HC regulates PPAR $\gamma$-mediated hepatic lipid metabolism through miR-130b-3p. Mol Ther Nucleic Acids. 2019;18:954-965. doi:10.1111/j.13652036.2010.04366.x

42. Chen LP, Wang H, Zhang Y, et al. Robust analysis of novel mRNA-lncRNA cross talk based on ceRNA hypothesis uncovers carcinogenic mechanism and promotes diagnostic accuracy in esophageal cancer. Cancer Manag Res. 2019;11:347-358. doi:10.2147/ cmar.s183310

43. Chen L, Li C, Peng Z, Zhao J, Gong G, Tan D. miR-197 expression in peripheral blood mononuclear cells from Hepatitis B virus-infected patients. Gut Liver. 2013;7(3):335-342. doi:10.5009/gnl.2013.7.3.335

44. Malone D, Karolin F, Ola W, Sandberg JK, Barbour JD. The dynamic relationship between innate immune biomarkers and interferon-based treatment effects and outcome in Hepatitis $\mathrm{C}$ virus infection is altered by telaprevir. PLoS One. 2014;9(8):e105665. doi:10.1371/journal. pone. 0105665

45. Zhang L, Zhou X, Dai Y, Lv C, Huang B. Establishment of interleukin time-resolved fluorescence immunoassay and its preliminary application in liver disease. J Clin Lab Anal. 2021;30:e23758.

\section{Publish your work in this journal}

Diabetes, Metabolic Syndrome and Obesity: Targets and Therapy is an international, peer-reviewed open-access journal committed to the rapid publication of the latest laboratory and clinical findings in the fields of diabetes, metabolic syndrome and obesity research. Original research, review, case reports, hypothesis formation, expert opinion and commentaries are all considered for publication. The manuscript management system is completely online and includes a very quick and fair peer-review system, which is all easy to use. Visit http://www.dovepress.com/testimonials.php to read real quotes from published authors.

Submit your manuscript here: https://www.dovepress.com/diabetes-metabolic-syndrome-and-obesity-targets-and-therapy-journal 